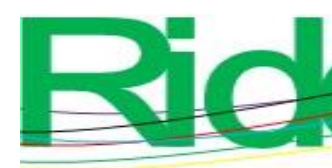

\title{
Guía de enseñanza para el aprendizaje exitoso en educación a distancia universitaria
}

\author{
Teaching Methods for Successful Learning in University Distance Education
}

Guia de ensino para aprendizagem bem-sucedida em educação a distância universitária

Elvira Ivone González Jaimes

Universidad Autónoma del Estado de México, México

ivonegj@hotmail.com

http://orcid.org/0000-0002-5328-5586

\section{Resumen}

El objetivo de este estudio fue analizar las estrategias exitosas de aprendizaje usadas por estudiantes universitarios para adquirir conocimiento en el Portal de la Plataforma Educativa (Seduca3) del nivel superior en educación a distancia, perteneciente a la Universidad Autónoma del Estado de México (UAEM). Para ello, se realizó un diseño no experimental de tipo documental y de campo con corte trasversal y método mixto (cuantitativo y cualitativo). El instrumento usado fue aplicado a 165 estudiantes con aprendizajes significativos de cinco carreras universitarias correspondientes a las áreas de ciencias económicas administrativas y ciencias biológicas. Con esto se procuró obtener estándares exitosos de enseñanza para ofrecer a los diseñadores de plataformas educativas guías para homologar y facilitar el aprendizaje. Los resultados demuestran que las estrategias que llevan al éxito académico son las siguientes: 1) conocimiento de objetivos y criterios de evaluación (qué y para qué se va a aprender); 2) control del contexto (con qué se va a aprender); 3) atribuciones externas para el aprendizaje (cómo se va a aprender), y 4) habilidades de interacción social y aprendizaje con compañeros (con quién se va a aprender). Esta guía facilita la recuperación de lo aprendido, expresado con la elaboración, y asociado con la 


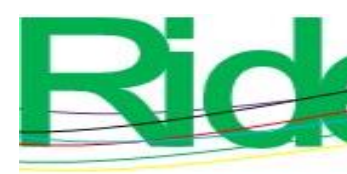

Revista Iberoamericana para la Investigación y el Desarrollo Educativo

ISSN $2007-7467$

retroalimentación positiva por logro, la cual genera la motivación intrínseca requerida para continuar aprendiendo. En pocas palabras, para promover un ciclo completo de aprendizaje significativo el estudiante debe tener claro "por qué, adónde, cómo, cuándo, cuánto, dónde y con quién" está aprendiendo. Por eso, se recomienda a los diseñadores de plataformas educativas que estos adverbios interrogativos aparezcan de forma explícita en los recursos brindados a los alumnos para facilitar y precisar la adquisición del conocimiento.

Palabras claves: aprendizaje significativo, educación a distancia, estrategias de enseñanza.

\section{Abstract}

The objective of study is to analyze the successful learning strategies to acquire knowledge in the Portal of the Educational Platform (SEDUCA3) of the higher level in distance education belonging to the Autonomous University of the State of Mexico (UAEM).Nonexperimental design of documentary and field type with transversal cut and mixed method (quantitative and qualitative). Applied to 165 students with significant learning in five university careers, covering the areas of administrative economics and biological sciences, because they wanted to observe the different ways of acquiring successful knowledge. To obtain successful teaching standards that will help the designers of educational platforms to standardize and facilitate learning.

The results show strategies that lead to academic success, which are: 1) Knowledge of objectives and evaluation criteria, indications of what and for what you will learn. 2) Control of the context, indications of what you are going to learn with; 3) External attributes for learning, exposing how you are going to learn. 4) Social interaction and peer learning skills, directing with whom you will learn. The key facilitates recovery of what has been learned, expressed through elaboration and associated with positive feedback by achievement. This is the generator of the intrinsic motivation required to continue learning. Showing a complete cycle of meaningful learning, where the student has clear the question adverbs "why, where, how, when, how much, where and with whom" he is learning. The contribution of the study is: clear and precise expression of the interrogative adverbs within the educational platforms and avoid masking them with synonyms, because they are keys of significant learning. The designers of educational platforms must include them in order to facilitate and specify the acquisition of knowledge.

Keywords: significant learning, distance education, teaching strategies. 


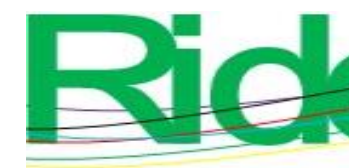

\section{Revista Iberoamericana para la Investigación y el Desarrollo Educativo ISSN 2007 - 7467}

\section{Resumo}

O objetivo deste estudo foi analisar as estratégias de aprendizagem bem-sucedidas utilizadas por estudantes universitários para adquirir conhecimentos no Portal da Plataforma Educacional (Seduca3) do nível superior em educação a distância, pertencente à Universidade Autônoma do Estado do México (UAEM). Para tanto, foi realizado um delineamento não experimental do tipo documental e de campo, com corte transversal e método misto (quantitativo e qualitativo). $\mathrm{O}$ instrumento utilizado foi aplicado a 165 alunos com aprendizagem significativa em cinco carreiras universitárias correspondentes às áreas de economia administrativa e ciências biológicas. Com isso, buscou-se obter padrões de ensino bem-sucedidos para oferecer aos projetistas de plataformas educacionais guias que padronizem e facilitem o aprendizado. Os resultados mostram que as estratégias que levam ao sucesso acadêmico são as seguintes: 1) conhecimento dos objetivos e critérios de avaliação (o que e para o que aprender); 2) controle de contexto (com o que aprender); 3) atribuições externas para aprender (como aprender) e 4) habilidades para interação social e aprendizagem entre pares (com quem aprender). Este guia facilita a recuperação do que foi aprendido, expresso na elaboração e associado ao feedback positivo para o aproveitamento, que gera a motivação intrínseca necessária para continuar aprendendo. Em suma, para promover um ciclo completo de aprendizagem significativa, o aluno deve ter clareza sobre "por que, onde, como, quando, quanto, onde e com quem” está aprendendo. Portanto, é recomendado aos projetistas de plataformas educacionais que esses advérbios interrogativos apareçam explicitamente nos recursos disponibilizados aos alunos para facilitar e especificar a aquisição de conhecimentos.

Palavras-chave: aprendizagem significativa, educação a distância, estratégias de ensino. Fecha Recepción: Mayo 2020 Fecha Aceptación: Octubre 2020 


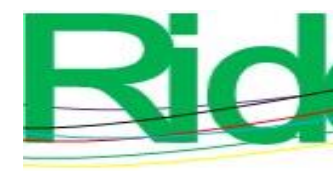

Revista Iberoamericana para la Investigación y el Desarrollo Educativo ISSN 2007 - 7467

\section{Introducción}

El objetivo de esta investigación consistió en medir y analizar las estrategias exitosas de aprendizaje usadas por estudiantes para adquirir conocimiento significativo en el portal de la plataforma educativa (Seduca3) del nivel superior en educación a distancia perteneciente a la Universidad Autónoma del Estado de México (UAEM). Esta iniciativa fue desarrollada con el propósito de recabar información para atender los problemas que afectan el rendimiento de la mayoría de los alumnos de la referida modalidad educativa, en la cual se reportan los siguientes datos: índice de reprobación (11.4 \% y $47 \%)$, índice de abandono académico (18.1\% y $32.2 \%$ ) e índices de eficiencia terminal (por corte: $11.5 \%$ y $41.7 \%$; global: $11 \%$ y $65 \%$ ) (UAEM, 2018).

Para tener un contexto amplio se evaluó a 165 estudiantes con altos rendimientos de cinco carreras profesionales, pertenecientes a dos áreas del conocimiento: ciencias económicas administrativas y ciencias biológicas, áreas supervisadas por la Dirección de Educación Continua y a Distancia de la UAEM e impartidas por Seduca3.

El instrumento utilizado fue el cuestionario de evaluación de las estrategias de aprendizaje de los estudiantes universitarios (Ceveapeu), ya que 1) está diseñado y validado con estudiantes universitarios de habla hispana y porque 2) es sensible para tamizar estrategias de aprendizaje desarrolladas dentro de plataformas educativas a través de veinticinco ítems focalizados en el uso, apoyo y control de material electrónico, así como en el procesamiento del conocimiento interactivo y en redes. Este instrumento, además, sirvió como sustento para contestar la siguiente pregunta de investigación: ¿cuáles estrategias de enseñanza orientan la adquisición de conocimiento significativo expresado en altos aprovechamientos académicos en educación a distancia?

\section{Educación a distancia}

En la actualidad, la educación a distancia se ha convertido en una herramienta didáctica indispensable para promover el aprendizaje. Esto ha sido posible gracias al empleo de las tecnologías de la información y la comunicación (TIC), las cuales han facilitado el "acceso universal a la educación, la igualdad en la instrucción, el ejercicio de la enseñanza y el aprendizaje de calidad y el desarrollo profesional de los docentes, así como a la gestión dirección y administración más eficientes del sistema educativo" (Organización de la Naciones Unidas para la Educación la Ciencia y la Cultura [Unesco], 2015, p. 23). 


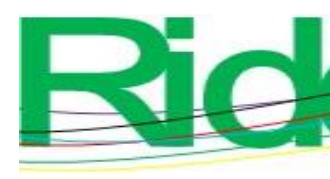

Revista Iberoamericana para la Investigación y el Desarrollo Educativo

ISSN 2007 - 7467

Según la Unesco (2013), con el uso de diversos dispositivos digitales se han podido conseguir grandes beneficios, entre los que se pueden subrayar los siguientes: 1) mayor alcance e igualdad de oportunidades en la educación, 2) facilidad para el aprendizaje personalizado, 3) respuesta y evaluación inmediatas, 4) aprendizaje en cualquier momento y lugar, 5) empleo productivo del tiempo pasado en el aula, 6) creación de nuevas comunidades de educandos, 7) apoyo al aprendizaje en lugares concretos, 8) mejora del aprendizaje continuo, 9) vínculo entre la educación formal y no formal, 10) mínimos trastornos para el aprendizaje en las zonas de conflicto y de desastre, 11) apoyo a los educandos con discapacidad, 12) mejora de la comunicación y la administración y 13) máxima eficacia en función de los costos.

Para conseguir tales beneficios, la educación a distancia se apoya en tres elementos fundamentales: 1) las TIC, las cuales permiten concretar su operación informativa, ya que son un conjunto de medios tecnológicos para la comunicación; 2) las tecnologías del aprendizaje y el conocimiento (TAC), que estimulan la alfabetización tecnológica y el aprendizaje significativo, pues son herramientas o medios didácticos (Shen, Wang y Shen, 2009) y 3) las tecnologías del empoderamiento y la participación (TEP), las cuales promueven el aprendizaje colaborativo, porque son las redes sociales del conocimiento formal e informal que permiten la construcción de la información (Dolors, 2019a). Estas tienen sus leyes éticas dirigidas al mundo virtual y funcionan a favor de la adquisición del conocimiento (Dolors, 2019b).

Ahora bien, a pesar de todas estas ventajas, se puede afirmar que en México la educación abierta o a distancia constituye solo $14 \%$ del total de la matrícula de educación superior en el país (Tuiran, 2019). Esto se debe a que desde el principio (hace 30 años) esta ha sido concebida únicamente como un suplemento de la educación presencial o como un método educativo para llegar a las poblaciones marginadas (sea por distancia, tiempo o dinero). En pocas palabras, no se toma en cuenta que la educación a distancia es una excelente opción para alfabetizar en torno al lenguaje tecnológico, requerimiento básico para promover la inclusión laboral y la cobertura de comunicación e información en todos los medios actuales.

La importancia que tiene este medio formativo se ve reflejada en la reforma educativa, capítulo XI, artículo 84, De las tecnologías de la información, comunicación, conocimiento y aprendizaje digital, donde se expone que la educación que imparta el Estado utilizará a las 


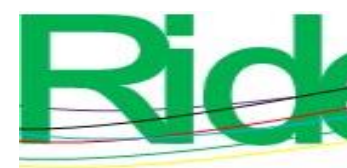

Revista Iberoamericana para la Investigación y el Desarrollo Educativo

ISSN $2007-7467$

TIC y el aprendizaje digital para fortalecer los modelos pedagógicos, la innovación educativa y para cerrar la brecha digital que se tiene con otros países desarrollados, estableciendo la agenda digital educativa que va a marcar la adaptación a los cambios tecnológicos, la innovación práctica y la creatividad para la resolución de problemas del desarrollo (Diario Oficial de la Federación [DOF], 2019).

\section{Modelo educativo no escolarizado a distancia en la UAEM}

El modelo educativo no escolarizado a distancia está diseñado para programas educativos formales de niveles medio superior, profesionales y avanzados. La Dirección de Educación Continua y a Distancia (Decyd) — desde 2004 - propone y supervisa el desarrollo de los programas de aprendizaje, los cuales presentan contenidos científicos y tecnológicos vigentes requeridos en el mercado laboral nacional e internacional con el fin de promover una formación integral y de calidad.

Asimismo, el Departamento de Estudios Profesionales del Sistema a Distancia diseña, coordina y evalúa los programas de enseñanza, así como las guías de estudio específicas para distintas asignaturas (UAEM, 2020). La plataforma educativa utilizada es el Portal de Servicios Educativo (Seduca3) - perteneciente a la UAEM-, la cual se ha venido perfeccionando y actualizando con la ejecución desde enero de 2015 de su versión 3. Esta se encarga de brindar los programas de enseñanza y materiales para operar las guías de estudio y las herramientas tecnológicas. Para ello, usa tecnología de cómputo a la vanguardia, certificada por la norma ISO 9001:2008 en desarrollo de actos académicos de educación continua y diseño gráfico de materiales educativos en línea (UAEM, 2020).

\section{Cuestionario de evaluación de las estrategias de aprendizaje de los estudiantes universitarios (Ceveapeu)}

Este cuestionario se fundamenta en el modelo de aprendizaje autorregulado de Pintrich y Schrauben (1992), el cual toma en cuenta el aprendizaje sostenible en educación, por lo que rebasa las primeras escalas reconocidas de evaluación de estrategias de aprendizaje, como son la escala de estrategias de aprendizaje (ACRA) (Román y Gallegos, 2001), la adaptación del cuestionario de estrategias de aprendizaje (CEA) (Beltrán, Pérez y Ortega, 2006) y la escala de estrategias de aprendizaje (ACRA-abreviada) (De la Fuente y Justicia, 2003). 


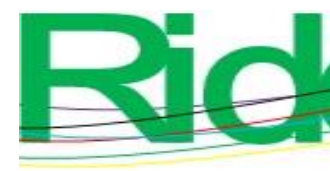

Revista Iberoamericana para la Investigación y el Desarrollo Educativo ISSN 2007 - 7467

El presente cuestionario evalúa las estrategias de aprendizaje en diferentes contextos, entre las que se pueden incluir las plataformas educativas, ya que se pueden adecuar a las nuevas herramientas electrónicas. De este modo se puede establecer la conjunción teórica de adquisición, fijación y reproducción del conocimiento en un modelo o "mapa" lo más completo posible, integrante de diversas estrategias sustantivas del aprendizaje (Gargallo, Suárez-Rodríguez y Pérez-Pérez, 2009).

El cuestionario presenta dos escalas: 1) estrategias afectivas, de apoyo control, constituidas por las subescalas de estrategias motivacionales, estrategias afectivas, estrategias de control del contexto, interacción social y manejo de recursos, así como estrategias metacognitivas, todas incluidas en 53 ítems con $\alpha=0.819$. Asimismo, 2) estrategias relacionadas con el procesamiento, que contienen las subescalas de búsqueda, recogida y selección de información, adquisición, elaboración, organización y almacenamiento, estrategias de personalización y creatividad, transferencia y uso de la información, todas incluidas en 35 ítems con $\alpha=0.864$ (Gargallo et al., 2009).

El cuestionario presenta adecuada validez de constructo, contrastada por la valoración de los jueces y por análisis factorial. La mayoría de las escalas muestran una correlación significativa al nivel 0.01 y confiabilidad general en alfa de Cronbach $(\alpha=0.841)$.

En total, contiene 88 ítems organizados en dos escalas, nueve subescalas y veinticinco estrategias. Además, posee los siguientes cinco niveles de calificación: muy adecuado (puntos 4), de acuerdo (3 puntos), indeciso ( 2 puntos), en desacuerdo ( 1 punto) y muy desadecuado (0 puntos), calificados por estos rangos: buenos, regulares y malos (Gargallo et al., 2009).

\section{Método}

La presente investigación ha sido desarrollada con base en un diseño no experimental de tipo documental y de campo, con corte trasversal y método mixto (cuantitativo y cualitativo), con estadística descriptiva e inferencial. Se ha aplicado a un grupo de alumnos con los más altos y significativos rendimientos académicos dentro de sus carreras profesionales (en concreto, cinco carreras profesionales pertenecientes a dos áreas de conocimiento: ciencias económicas administrativas y ciencias biológicas) con el fin de analizar las estrategias de aprendizaje exitosas en educación a distancia. La población estuvo 


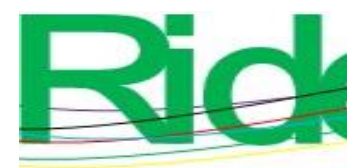

Revista Iberoamericana para la Investigación y el Desarrollo Educativo

ISSN 2007 - 7467

conformada por 716 estudiantes, mientras que la muestra se obtuvo con la fórmula para poblaciones finitas. De este modo se consiguió una muestra de 165 estudiantes $(23.05 \%)$.

\section{Procedimiento}

- Fase 0. Investigación documental, análisis de trayectorias educativas para seleccionar a estudiantes con aprendizaje significativo por sus altos promedios académicos en cada una de las cinco carreras profesionales.

- Fase 1. Promoción por correo electrónico y difusión ética del procedimiento. Se realizó una invitación personalizada a cada uno de los estudiantes; en esta se les informó que habían sido seleccionados por sus buenos promedios dentro de sus carreras universitarias, por lo que formarían parte de un proyecto de investigación registrado en la universidad. También se les solicitó su participación con la resolución del cuestionario de evaluación de las estrategias de aprendizaje de los estudiantes universitarios (Ceveapeu), facilitado a través de una página web. Igualmente, se les informó sobre el trato ético de sus datos y resultados, ambos con uso confidencial.

- Fase 2. Compilación y extracción de base de datos.

- Fase 3. Análisis de los datos: El análisis cuantitativo estuvo conformado por 1) la estadística descriptiva para datos socioeducativos y datos del cuestionario de evaluación de las estrategias de aprendizaje de los estudiantes universitarios (Ceveapeu); con esto se consiguieron medidas de la frecuencia, medias y porcentaje de edad, género, licenciatura, nivel académico y subescalas del cuestionario. Asimismo, 2) en la estadística inferencial se utilizó la prueba T de student para saber la existencia de diferencia entre cinco grupos. Para el análisis cualitativo se utilizó el método de análisis de proceso jerárquico, empleando la técnica de matriz de prioridad y consistencia en las subescalas de estrategias de aprendizaje asociadas a su alto rendimiento académico en su carrera profesional (Saaty, 2010).

\section{Resultados}

Empleando la estadística descriptiva, se conocieron las principales características de la muestra: igualdad de género en cuanto a participación, edad entre 23 y 24.11 años, $74.5 \%$ trabajan y cursan $4 .^{\circ}$ año de la carrera (tabla 1$)$. 


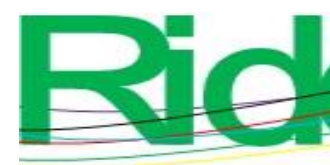

Revista Iberoamericana para la Investigación y el Desarrollo Educativo ISSN 2007 - 7467

Tabla 1. Características socioacadémicas y laborales de la muestra

\begin{tabular}{|c|c|c|c|c|c|}
\hline & & & \multicolumn{2}{|c|}{ Género } & \\
\hline Espacios académicos & Licenciatura & Muestra & Masculino & Femenino & Total \\
\hline \multirow{3}{*}{ Facultades } & Administración & 81.42 & 38 & 43 & 81 \\
\hline & $\begin{array}{l}\text { Informática } \\
\text { Administrativa }\end{array}$ & 28.98 & 17 & 12 & 29 \\
\hline & Enfermería & 20.47 & 6 & 15 & 21 \\
\hline \multirow[b]{2}{*}{ Unidades académicas } & Logística & 18.86 & 13 & 6 & 19 \\
\hline & $\begin{array}{l}\text { Negocios } \\
\text { Internacionales }\end{array}$ & 14.95 & 8 & 7 & 15 \\
\hline Total & & 164.68 & 82 & 83 & 165 \\
\hline & & \multicolumn{2}{|c|}{ Trabajan } & & \\
\hline \multicolumn{2}{|c|}{ Edad cronológica } & SÍ & $\mathrm{NO}$ & \multicolumn{2}{|c|}{ Año académico } \\
\hline 18 a 20 años & 28 & 12 & 16 & $1 .^{\circ}$ & 12 \\
\hline 21 a 22 años & 49 & 28 & 21 & $2 .^{\circ}$ & 32 \\
\hline 23 a 24 años & 51 & 38 & 13 & $3 .^{\circ}$ & 46 \\
\hline 24 a 26 años & 26 & 16 & 10 & $4 .^{\circ}$ & 65 \\
\hline 27 a más años & 11 & 9 & 2 & $5 .^{\circ}$ & 10 \\
\hline Total & 165 & 103 & 62 & & 165 \\
\hline
\end{tabular}

Fuente: Elaboración propia

En la prueba T de student para cinco muestras independientes no se observó diferencia significativa entre las medias y la desviación estándar de los cinco grupos, por lo que se asume que tienen una distribución normal (tabla 2).

Para el estudio cualitativo se utilizó el método de análisis de proceso jerárquico, específicamente la técnica de matriz de prioridad y consistencia en las subescalas de estrategias de aprendizaje asociadas a su aprendizaje significativo por alto rendimiento académico en su carrera profesional (Saaty, 2010). En primer lugar, se observó el Conocimiento de objetivos y criterios de evaluación; en segundo lugar, se percibió el Control del contexto; en tercer lugar, se analizaron las Atribuciones externas; en cuarto lugar, se tomaron en cuenta las Habilidades de interacción social y aprendizaje con compañeros, y por último se incluyeron 1) la Elaboración y 2) la Motivación intrínseca (tabla 2). 
Tabla 2. Descriptivos y jerarquía de las estrategias de aprendizaje

\begin{tabular}{|c|c|c|c|c|c|}
\hline Escalas & Subescalas & Estrategias & Media & D.S & Lugar \\
\hline \multirow{15}{*}{$\begin{array}{l}\text { Estrategias } \\
\text { afectivas, de } \\
\text { apoyo y } \\
\text { control (o } \\
\text { automanejo) } \\
(\alpha=.819) \\
\text { (53 ítems) }\end{array}$} & \multirow{7}{*}{$\begin{array}{l}\text { Estrategias } \\
\text { motivacionales } \\
\quad(\alpha=.864) \\
(20 \text { ítems })\end{array}$} & $\begin{array}{l}\text { Motivación intrínseca }(\alpha= \\
.500)(3 \text { items })\end{array}$ & 11.45 & 0.55 & 5 \\
\hline & & $\begin{array}{l}\text { Motivación extrínseca }(\alpha= \\
.540)(2 \text { ítems })\end{array}$ & 7.45 & 0.68 & \\
\hline & & $\begin{array}{l}\text { Valor de la tarea }(\alpha=.692) \\
(4 \text { ítems) }\end{array}$ & 13.12 & 2.88 & \\
\hline & & $\begin{array}{l}\text { Atribuciones internas }(\alpha= \\
.537) \text { (3 ítems) }\end{array}$ & 10.98 & 1.02 & \\
\hline & & $\begin{array}{l}\text { Atribuciones externas }(\alpha= \\
.539)(2 \text { items })\end{array}$ & 7.48 & 0.52 & 3 \\
\hline & & $\begin{array}{l}\text { Autoeficacia y expectativas } \\
(\alpha=.743) \text { (4 ítems) }\end{array}$ & 15.01 & 0.99 & \\
\hline & & $\begin{array}{l}\text { Concepción de la } \\
\text { inteligencia como } \\
\text { modificable }(\alpha=.595) \\
(2 \text { ítems }) \\
\end{array}$ & 6.29 & 1.71 & \\
\hline & \multirow{2}{*}{$\begin{array}{c}\text { Componentes } \\
\text { afectivos }(\alpha=.707) \\
\text { (8 ítems) }\end{array}$} & $\begin{array}{l}\text { Estado físico y anímico }(\alpha= \\
.735)(4 \text { ítems })\end{array}$ & 14.82 & 1.18 & \\
\hline & & $\begin{array}{l}\text { Ansiedad }(\alpha=.714)(4 \\
\text { ítems) }\end{array}$ & 14.23 & 1.77 & \\
\hline & \multirow{4}{*}{$\begin{array}{l}\text { Estrategias } \\
\text { metacognitivas } \\
(\alpha=.738) \\
(15 \text { ítems })\end{array}$} & $\begin{array}{l}\text { Conocimiento de objetivos y } \\
\text { criterios de evaluación }(\alpha= \\
.606)(2 \text { items })\end{array}$ & 7.67 & 0.33 & 1 \\
\hline & & $\begin{array}{l}\text { Planificación }(\alpha=.738) \text { (4 } \\
\text { ítems) }\end{array}$ & 15.32 & 0.68 & \\
\hline & & $\begin{array}{l}\text { Autoevaluación }(\alpha=.521)(3 \\
\text { ítems })\end{array}$ & 11.29 & 0.71 & \\
\hline & & $\begin{array}{l}\text { Control, autorregulación }(\alpha= \\
.660)(6 \text { ítems })\end{array}$ & 23.12 & 0.88 & \\
\hline & \multirow{2}{*}{$\begin{array}{c}\text { Estrategias de } \\
\text { control del contexto, } \\
\text { interacción social y } \\
\text { manejo de recursos } \\
(\alpha=.703) \\
(10 \text { ítems })\end{array}$} & $\begin{array}{l}\text { Control del contexto }(\alpha= \\
.751)(4 \text { items })\end{array}$ & 15.63 & 0.37 & 2 \\
\hline & & $\begin{array}{l}\text { Habilidades de interacción } \\
\text { social y aprendizaje con } \\
\text { compañeros }(\alpha=.712)(6 \\
\text { ítems) }\end{array}$ & 23.47 & 0.53 & 4 \\
\hline \multirow{4}{*}{$\begin{array}{c}\text { Estrategias } \\
\text { relacionadas } \\
\text { con el } \\
\text { procesamiento } \\
\text { de la } \\
\text { información } \\
(\alpha=.864) \\
\text { (35 ítems) }\end{array}$} & \multirow{2}{*}{$\begin{array}{l}\text { Estrategias de } \\
\text { búsqueda y } \\
\text { selección e } \\
\text { información } \\
(\alpha=.705) \\
(8 \text { ítems })\end{array}$} & $\begin{array}{l}\text { Conocimiento de fuentes y } \\
\text { búsqueda de información } \\
(\alpha=.685) \text { (4 ítems) }\end{array}$ & 15.01 & 0.99 & \\
\hline & & $\begin{array}{l}\text { Selección de información } \\
(\alpha=.630) \text { (4 ítems) }\end{array}$ & 14.87 & 1.13 & \\
\hline & \multirow{2}{*}{$\begin{array}{l}\text { Estrategias de } \\
\text { procesamiento y uso } \\
\text { de la información }\end{array}$} & $\begin{array}{l}\text { Adquisición de información } \\
(\alpha=.677) \text { (3 ítems })\end{array}$ & 10.34 & 1.66 & \\
\hline & & $\begin{array}{l}\text { Elaboración }(\alpha=.739)(4 \\
\text { ítems })\end{array}$ & 15.45 & 0.55 & 5 \\
\hline
\end{tabular}




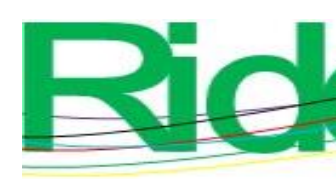

Revista Iberoamericana para la Investigación y el Desarrollo Educativo ISSN 2007 - 7467

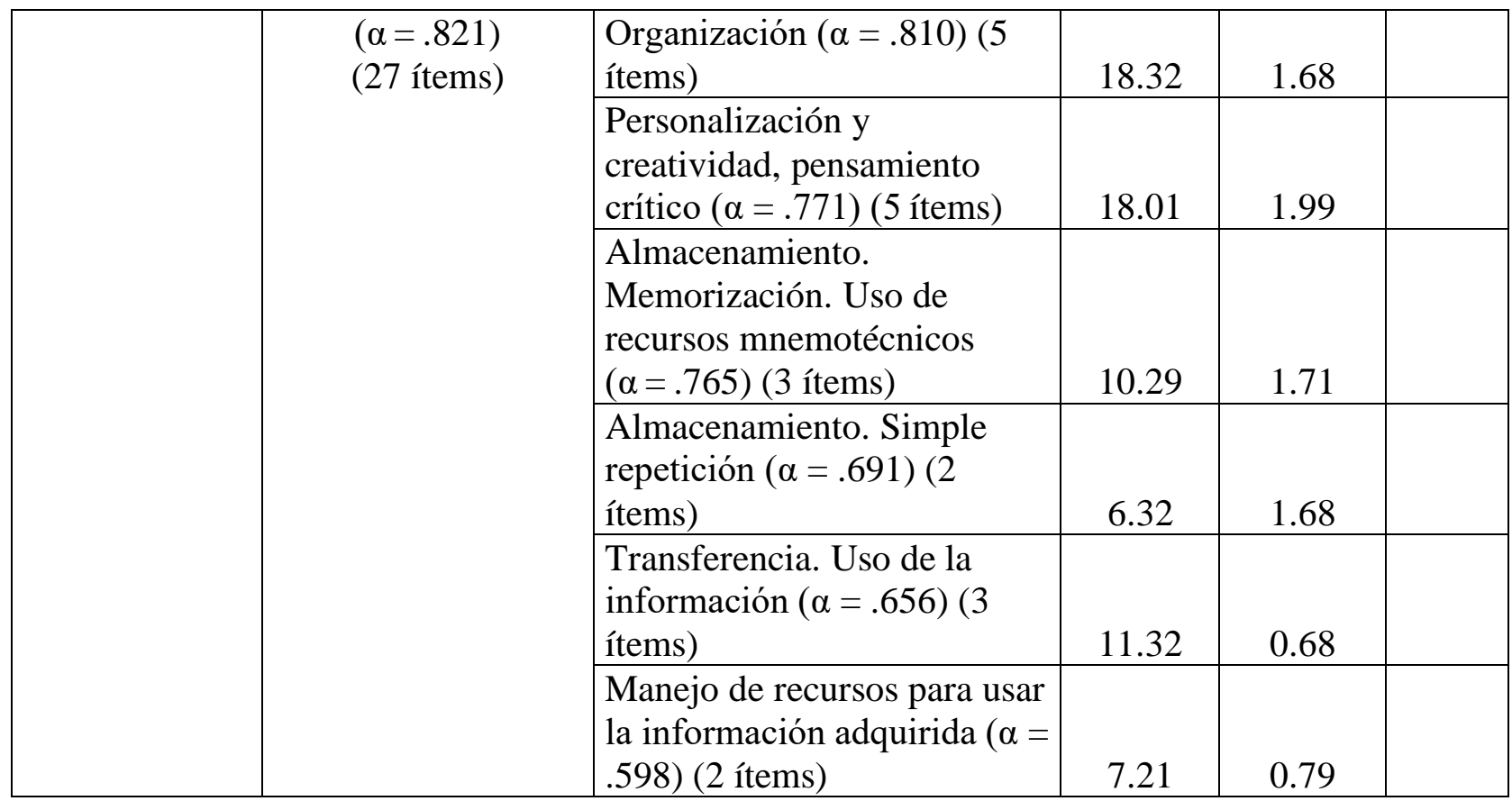

Fuente: Elaboración propia

\section{Discusión}

La educación a distancia tiene específicas condiciones de enseñanza. Se debe tener claro que aun cuando la interactividad es una de las principales características de las plataformas virtuales, su simple uso no mejora el aprendizaje ni su calidad, ya que su impacto se asocia a las actitudes que muestren los usuarios (González, López, Trujillo y Rojas, 2018; Villalba, García, Gaxiola y Borbolla, 2017). Por eso, es necesario enfocar los esfuerzos en el diseño de estrategias de aprendizaje que permitan atender el tipo de dificultades en la adquisición, almacenamiento y recuperación de la información, ya que son la base del aprendizaje autorregulado, es decir, de la manera en que el individuo es capaz de ajustar sus acciones y metas al ambiente tecnológico de la plataforma educativa (Moreno, 2017). La capacidad de autorregulación, en pocas palabras, juega un papel clave en el éxito académico y en cualquier contexto virtual (Navarrete y Manzanilla, 2017), pues le brinda al usuario una sensación de adaptación ante las actividades presentadas (González et al., 2018).

Por otra parte, se puede afirmar que los antecedentes teóricos se ven reflejados en los resultados de la presente investigación. Es decir, en primer lugar, se encuentra el Conocimiento de objetivos y criterios de evaluación, lo que significa que el aprendiz debe de tener claro qué y para qué va a aprender. En segundo lugar, se tiene el Control del contexto, lo que se asocia al criterio con qué va a aprender, para lo que se requiere conocimiento y 


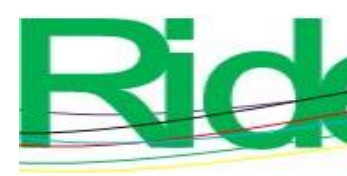

Revista Iberoamericana para la Investigación y el Desarrollo Educativo

ISSN $2007-7467$

manejo de los dispositivos o herramientas empleadas. En tercer lugar, se observan las Atribuciones externas para el aprendizaje, esto es, las estrategias de aprendizaje utilizadas para la adquisición de los conocimientos. En cuarto lugar, se muestran las Habilidades de interacción social y aprendizaje con compañeros, lo que refleja otras habilidades de adquisición con quién va a aprender, apoyándose en la interacción social. En quinto lugar, se aprecian dos estrategias: a) la Elaboración o el proceso de recuperación de lo aprendido, el cual se vincula con b) la Motivación intrínseca o retroalimentación de lo aprendido. La unión de ambas estrategias de aprendizaje confirma el cierre de un ciclo y la apertura de otro para continuar aprendiendo (Pando, 2018). En otras palabras, los resultados demuestran que no se deben perder de vista los elementos indispensables para que el aprendizaje sea exitoso.

Por otra parte, los aspectos teóricos deben estar vinculados con la autorregulación, la cual se potencia cuando los estudiantes saben con certeza "por qué, adónde, cómo, cuándo, cuánto, dónde y con quién" se está aprendiendo, criterios que algunas veces son obviadas por los diseñadores de las plataformas educativas. Por ello, para que se genere el sentimiento de fluidez y confort del aprendizaje a distancia los diseñadores deben facilitar la información con base en guías de pensamiento que tomen en cuenta los referidos adverbios interrogativos. Así se pueden disminuir los errores en la adquisición, asimilación y reproducción de la información que se quiere enseñar y acelera la fluidez del conocimiento (Csikszentmihalyi, 2014).

\section{Conclusiones}

En el proceso de educación a distancia se debe procurar el entrenamiento con guías de aprendizaje claras que faciliten la adaptación y el acceso a la información suministrada. En otras palabras, no se debe perder de vista que el estudiante virtual se encuentra "solo" frente a una pantalla que por sí misma no garantiza una comunicación eficaz. Por eso, trabajar en el empoderamiento de las herramientas electrónicas le va a permitir al alumno desenvolverse en dicho medio con la misma naturalidad que se genera en el entorno físico del aula. En pocas palabras, cuando se ofrecen guías de pensamiento claras se puede agilizar la adquisición del conocimiento, independientemente de la carrera o área de estudio cursada. Por ende, sería conveniente que los diseñadores de las plataformas educativas desarrollaran las herramientas didácticas digitales tomando en consideración los interrogativos antes mencionados (por qué, adónde, cómo, cuándo, cuánto, dónde y con quién se está 


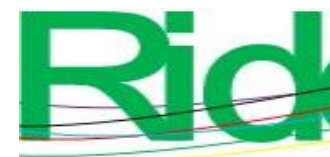

Revista Iberoamericana para la Investigación y el Desarrollo Educativo

ISSN $2007-7467$

aprendiendo), los cuales deben aparecer de forma explícita en los recursos que se les brindan a los alumnos. Esto, en definitiva, pudiera contribuir a la disminución de los índices de reprobación y abandono académico.

\section{Referencias}

Beltrán, J. A., Pérez, L. F. y Ortega, M. I. (2006). Cuestionario de estrategias de aprendizaje, CEA. Madrid: TEA.

Csikszentmihalyi, M. (2014). Flow and the Foundations of Positive Psychology Mihaly Csikszentmihalyi. The Collected Works of Mihaly Csikszentmihalyi. New York London. Springer Dordrecht Heidelberg. Doi: 10.1007/978-94-017-9088-8

De la Fuente, A. J. y Justicia, J. F. (2003). Escalas de estrategias de aprendizaje ACRAAbreviada para alumnos universitarios. Revista Electrónica de Investigación Psicoeducativa y Psicopedagógica, 1(2), 138-158. Recuperado de www.uned.es/revistaestilosdeaprendizaje/...7/.../lsr_7_abril_2011.pdf

Diario Oficial de la Federación [DOF] (2019). Nueva reforma educativa. Diario Oficial de la Federación, 30 de septiembre de 2019. México: Secretaría de Gobernación. Recuperado de http://www.diputados.gob.mx/LeyesBiblio/ref/lge/LGE_orig_30sep19.pdf

Dolors, R. H. (2019a). Optimizando nuestros cerebros para internet. Cuadernos de Pedagogía, (500), 168-170. Recuperado de https://dialnet.unirioja.es/servlet/autor?codigo=701093

Dolors, R. H. (2019b). Necesitamos un juramento hipocrático para tecnólogos. Cuadernos de Pedagogía, (502), 122-123. Recuperado de https://dialnet.unirioja.es/servlet/articulo?codigo=7114399

Gargallo, B., Suárez-Rodríguez, J. y Pérez-Pérez, C. (2009). El cuestionario CEVEAPEU. Un instrumento para la evaluación de las estrategias de aprendizaje de los estudiantes universitarios. Revista Electrónica de Investigación y Evaluación, 15, (2), 1-31. Recuperado de http://www.uv.es/RELIEVE/v15n2/RELIEVEv15n2_5.htm

González J. E., López, C. A., Trujillo, M.V. y Rojas, H. J. (2018). Estrategia didáctica de enseñanza y aprendizaje para programadores de software. Revista Interamericana para la Investigación y el Desarrollo Educativo, 9(17), 688-712. Doi: 10.23913/ride.v9i17.402 


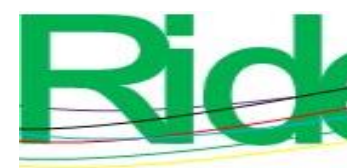

Revista Iberoamericana para la Investigación y el Desarrollo Educativo

ISSN $2007-7467$

Moreno, M. (2017). Educación a distancia universitaria en el despertar del siglo XXI. La educación a distancia en México: una década de sostenido esfuerzo institucional. Experiencias y perspectivas. México: Universidad Autónoma de Guadalajara.

Pintrich, P.R. y Schrauben, B. (1992). Student's Motivational Beliefs and their Cognitive Engagement in Classroom academic Tasks. En D.H. Schunck y J. Meece (Eds.), Students Percepcions in the Classrrom, 149-183. Hillsdale, N.J.: Lawrence Erlbaum. Navarrete, Z. y Manzanilla, G. H. (2017). Panorama de la educación a distancia en México. Revista Latinoamericana de Estudios Educativos, 13(1), 65-82. Recuperado de https://www.redalyc.org/pdf/1341/134152136004.pdf

Organización de la Naciones Unidas para la Educación la Ciencia y la Cultura [Unesco] (2013). Directrices de la UNESCO para las políticas del aprendizaje móvil. París: Organización de las Naciones Unidas para la Educación, la Ciencia y la Cultura. Recuperado de http://unesdoc.unesco.org/images/0021/002196/219662S.pdf

Organización de la Naciones Unidas para la Educación la Ciencia y la Cultura [Unesco] (2015). Educación 2030: Declaración de Incheon y marco de acción para la realización del objetivo de desarrollo sostenible 4. París: Organización de las Naciones Unidas para la Educación, la Ciencia y la Cultura. Recuperado de https://unesdoc.unesco.org/ark:/48223/pf0000245656_spa

Pando, V. F. (2018). Tendencias didácticas de la educación virtual: un enfoque interpretativo. Propósitos y Representaciones, 6(1), 463-505. Doi: http://dx.doi.org/10.20511/pyr2018.v6n1.167

Román, J. M. y Gallegos, S. (2001). Escalas de estrategias de aprendizaje (ACRA). México: Manual Moderno.

Saaty, L. T. (2010). El proceso analítico de jerarquía: planificación, establecimiento de prioridades, asignación de recursos (5. ${ }^{\mathrm{a}}$ ed.). México: McGraw-Hill.

Shen, L., Wang, M. and Shen, R. (2009). Affective e-Learning: Using "Emotional” Data to Improve Learning in Pervasive Learning Environment. Educational Technology \& Society, 12(2), 176-189. Recuperado de https://pdfs.semanticscholar.org/563e/3f5ead81b9491c7622695611a44b8fa9870d.p df 


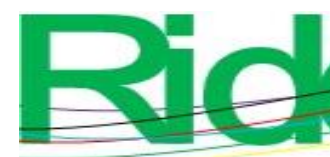

Tuiran, G. R. (2019). La educación superior: promesas de campaña y ejercicio de gobierno. Revista de Educación Superior, 48(190), 113-183. Recuperado de http://resu.anuies.mx/ojs/index.php/resu/article/view/715

Universidad Autónoma del Estado de México [UAEM] (2018). Agenda estadística. Secretaría de Planeación y Desarrollo Institucional, Toluca. Universidad Autónoma del Estado de México. Recuperado de http://planeacion.uaemex.mx/docs/AE/2018/AE2018.pdf

Universidad Autónoma del Estado de México [UAEM] (2020). Dirección de Educación Continua y a Distancia: Quiénes somos. Recuperado de https://campusvirtual.uaemex.mx/conocenos/\#somos

Villalba, R. S., García, G. T., Gaxiola, C. S. y Borbolla, I. J. (2017). Experiencia de enseñanza-aprendizaje con la plataforma virtual en la facultad de Medicina Veterinaria y Zootecnia de la Universidad Autónoma de Sinaloa. Revista de Investigación en Tecnologías de la Información (RITI), 5(10), 48-53. Recuperado de http://www.riti.es/ojs2018/inicio/index.php/riti/article/view/63 